\title{
Los docentes de Ciencias Sociales y la enseñanza de la Historia. Una mirada comparativa entre sus concepciones y las de sus estudiantes 1
}

\author{
Nilson Javier IBAGÓN-MARTÍN
}

Datos de contacto:

Nilson Javier lbagón-Martín Docente del Departamento de Historia de la Universidad del Valle, Colombia. nilson.ibagon@correounivalle.edu.co
Recibido: $17 / 06 / 2020$

Aceptado: $26 / 11 / 2020$

\begin{abstract}
RESUMEN
El presente texto socializa los resultados de un estudio comparativo orientado a identificar las concepciones de un grupo de docentes de ciencias sociales colombianos $(n=61)$ y sus estudiantes $(n=764)$, alrededor de las prácticas de enseñanza de la historia, los objetivos formativos de estas y el interés de aprendizaje sobre determinados contenidos históricos del alumnado. La investigación se basó en el paradigma cuantitativo. El diseño ha sido no experimental de carácter descriptivo e inferencial. Se empleó un cuestionario cerrado cuyos ítems de respuestas debían ser valorados por medio de una escala Likert. Luego, la información obtenida a través de este instrumento se trianguló con el análisis de las planeaciones institucionales de los centros educativos en los que laboran los profesores participantes. Los resultados en los tres núcleos de indagación evidencian diferencias significativas entre las concepciones de los docentes y sus estudiantes, así como entre lo manifestado por los profesores y lo registrado en las planeaciones. Estas diferencias dejan de manifiesto una serie de problemáticas que afectan negativamente los alcances formativos de la historia entendida como saber escolar. Las incongruencias y vacíos identificados entre las concepciones de los docentes y sus estudiantes, ratifican la necesidad de pensar procesos de formación inicial y permanente del profesorado de Ciencias sociales en el campo específico de la didáctica de la historia.
\end{abstract}

PALABRAS CLAVE: Enseñanza de la Historia; práctica docente; aprendizaje histórico; Didáctica de la Historia; Colombia

\footnotetext{
${ }^{1}$ Artículo vinculado al Proyecto (PGC2018-094491-B-C33) financiado por el Ministerio de Ciencia e Innovación. Gobierno de España.
} 


\title{
The Teachers of social sciences and the teaching of history. A comparative look between their conceptions and those of their students
}

\begin{abstract}
This text socializes the results of a comparative study aimed at identifying the conceptions of a group of Colombian social science teachers $(n=61)$ and their students $(n=764)$, around the teaching practices of history, the formative objectives of these and the interest of learning about certain historical contents of the students. The research was based on the quantitative paradigm. The design has been non-experimental, descriptive and inferential. A closed questionnaire was used whose response items had to be assessed by means of a Likert scale. The information obtained through this instrument was triangulated with the analysis of the institutional plans of the educational centers in which the participating teachers work. The results in the three nuclei of inquiry show significant differences between the conceptions of the teachers and their students, as well as between what the teachers stated and what was recorded in the planning. These differences reveal a series of problems that negatively affect the educational scope of history understood as school knowledge. The inconsistencies and gaps identified between the conceptions of teachers and their students confirm the need to think about processes of initial and permanent training for teachers of social sciences in the specific field of didactics of history.
\end{abstract}

KEYWORDS: History teaching; Teaching practice; historical learning; Didactics of history; Colombia.

\section{Introducción}

A partir de la crítica de la idea de "integración" curricular que durante las últimas cuatro décadas ha sustentado las normativas oficiales de enseñanza de las Ciencias Sociales expedidas en Colombia, algunos investigadores han sostenido que la Historia fue invizibilizada y diluida como saber escolar en este país, dejando de ser, por lo tanto, enseñada en la básica primaria y secundaria (Acevedo \& Samacá, 2012; Guerrero, 2011; Vargas, Acosta \& Sánchez, 2013). Sin embargo, este tipo de postulados han comenzado a ser rebatidos a través de evidencia empírica que demostraría la influencia que aún mantiene la Historia en: las evocaciones en torno a las clases de Ciencias Sociales de los estudiantes recién graduados del bachillerato (Arias, 2015, 2017); la estructura y tipo de contenidos de los textos escolares de esta área (Ibagón, 2019; Padilla \& Bermúdez, 2016); la marcada presencia de 
contenidos históricos organizados cronológicamente en los planes de estudio de futuros profesores de Ciencias Sociales, (Aguilera, 2017; Ibagón, 2016; Rodríguez, 2013); las representaciones sociales de estos últimos en torno a su enseñanza (González, Santisteban \& Pagès, 2020), y; los proyectos de intervención educativa que adelantan algunos profesores en ejercicio (Gómez, 2015).

No obstante, salvo contadas excepciones, esta influencia ha sido asociada con discursos educativos y resultados de aprendizaje que poco o nada tienen que ver con el desarrollo del pensamiento histórico de los estudiantes, y que, por el contrario, profundizan procesos de transmisión de narrativas históricas lineales acríticas y desconectadas de las experiencias vitales de los educandos. En este sentido, las limitantes para pensar la enseñanza de la historia en Colombia no estarian definidas exclusivamente "(...) a partir de la presencia o no de la historia como asignatura independiente y autónoma en los planes de estudio, tal y como ingenuamente han creído algunos legisladores y académicos, sino en el tipo de prácticas que han y siguen fundamentando directamente su conocimiento y presencia en las instituciones educativas" (Ibagón \& Minte, 2019, p. 110).

En el marco de la caracterización y comprensión de esta problemática es clave, por lo tanto, no solo el análisis de las normativas curriculares, sino, a la vez, entender las lógicas particulares que orientan la práctica docente y su recepción por parte del estudiantado. De ahí que, en el presente estudio, reconociendo la importancia que tiene para la didáctica de la Historia comprender las ideas sobre la disciplina y su enseñanza por parte de docentes en ejercicio y en formación (Borries, 2011; Cerri, 2018; Gago, 2016, 2018; González, 2018; McCully, 2012; Monteagudo, Molina \& Miralles, 2015; Voet \& De Wever, 2016; Yilmaz, 2008), y alumnos (Angvik \& Borries, 1997; Barca, 2019; Gago, 2016; Kitson, Steward, \& Husbands, 2015; Miralles, Prats $\&$ Tatjer, 2012; Schmidt, 2019), se haya definido como objetivo, identificar las diferencias y similitudes entre las concepciones de un grupo de profesores de ciencias sociales y sus estudiantes, alrededor de las prácticas de enseñanza de la historia, los objetivos formativos que orientan dichas clases $\mathrm{y}$, el reconocimiento de los intereses de aprendizaje en torno a determinados contenidos históricos.

A través de esta comparación, se realiza una reflexión sobre las dificultades que se presentan en la enseñanza de la historia al interior de las aulas de clase y, los retos que existen en la formación inicial y permanente de docentes de Ciencias Sociales en Colombia, respecto al campo específico de la didáctica de la historia. 


\section{Método}

La investigación se basó en el paradigma cuantitativo. El diseño ha sido no experimental de carácter descriptivo e inferencial. En el estudio participaron docentes de educación básica secundaria y media que, en el año 2019, impartían el área de Ciencias Sociales en trece instituciones educativas de carácter público $(n=8)$ y privado $(n=5)$ ubicadas en la ciudad de Bogotá, Colombia. En estas instituciones, previamente, se había realizado un proceso investigativo en torno al aprendizaje de la historia y el desarrollo de la conciencia histórica, dirigido a estudiantes del último grado de educación obligatoria $(n=764)^{2}$; en dicho estudio, se empleó un instrumento de recolección de información similar al utilizado posteriormente con los docentes.

El número de participantes se calculó tomando como referencia, el total de docentes que en cada institución educativa impartian en secundaria clases de Ciencias Sociales -Incluyendo Ciencia Política y Economía- $(\mathrm{n}=67)$. Al aplicar criterios de medición que aseguraran la representatividad de la muestra $-99 \%$ de confianza y $5 \%$ de error-, esta, quedó conformada por 61 docentes, de los cuales, el 59\% eran hombres $(n=36)$ y el $41 \%$ mujeres $(n=25)$. Un porcentaje importante de estos docentes estaban en rangos de edad entre los 31 a 35 años $(32,8 \%), 36$ a 40 años $(27,9 \%)$, y 41 a 45 años $(13,1 \%)$, mientras que los rangos comprendidos entre los 25 a 30 años $(9,8 \%), 46-50$ años (4,9\%), 51-55 años $(8,2 \%), 56$ a 60 años $(1,6 \%)$ y $61-65$ años $(1,6 \%)$ registraron los menores porcentajes. La media de edad de los participantes fue de 37, 5 años.

En cuanto a su formación de base, el 85,2\% $(n=52)$ contaba con títulos asociados a licenciaturas de Ciencias Sociales, y, el 14,8\% restante tenía títulos profesionales de Historia $(n=4,-6,6 \%-)$, Sociología $(n=3,-4,9 \%-)$ y Geografia ( $\mathrm{n}=2,-3,3 \%-)$. A nivel de estudios de posgrado, 25 docentes tenían título de Maestría (41\%) -en su mayoría de educación, pedagogía y docencia $(\mathrm{n}=19)-$, y 12 eran especialistas $(19,8 \%)$.

El instrumento de recolección de información principal se basó en un cuestionario cerrado empleado en investigaciones llevadas a cabo en el Mercosur sobre los jóvenes y su relación con la historia, en las cuales, participaron estudiantes y profesores de Argentina, Brasil, Chile, Uruguay y Paraguay (Amézola \& Cerri, 2018; Cerri 2018). El cuestionario fue adaptado y validado -juicio de expertos y estudio piloto- para efectos

\footnotetext{
${ }^{2}$ Los datos obtenidos en este estudio hacen parte de la investigación doctoral del autor titulada "Aprendizaje histórico en estudiantes colombianos y españoles. Análisis comparativo a partir de sus concepciones sobre la Historia", la cual se lleva a cabo en la Universidad de Murcia-España.
} 
del desarrollo de una investigación doctoral centrada en el análisis de las concepciones de estudiantes colombianos y españoles en torno a la historia. Finalizado el proceso de recolección de información con los estudiantes colombianos, se consideró pertinente en el mismo año, realizar un segundo estudio que indagara las posiciones de sus profesores frente a cuestiones que hacian parte de uno de los cinco núcleos de indagación del instrumento base que les fue aplicado: Cultura escolar -enseñanza y aprendizaje de la historia-. En este sentido, se retomaron tres preguntas acompañadas de una serie de ítems respuestas cerradas-que debían ser valorados por los docentes de acuerdo a una escala Likert de cinco puntos (Tabla 1). En las dos primeras preguntas - ¿Qué ocurre normalmente en sus clases de Historia? Y ¿En qué se concentran más sus clases de Historia? - la escala iba de "nunca" -valor uno- a "siempre" -valor cinco-. En la tercera pregunta - ¿Cuál de estos temas les interesan más a los estudiantes? La escala iba de "muy poco" -valor uno- a "interesa mucho" -valor cinco-.

Tabla 1.

Preguntas e items analizados

\begin{tabular}{|c|c|}
\hline Preguntas & Ítems \\
\hline $\begin{array}{l}\text { ¿Qué ocurre normalmente en sus clases } \\
\text { de Historia? }\end{array}$ & $\begin{array}{l}\text { 1.Los estudiantes oyen mis explicaciones } \\
\text { sobre el pasado. } \\
\text { 2.Informo a los estudiantes sobre lo que } \\
\text { fue bueno o malo, verdadero o falso en la } \\
\text { historia. } \\
\text { 3.Discutimos diferentes explicaciones } \\
\text { sobre lo que aconteció en el pasado. } \\
\text { 4.Los estudiantes analizan diversas } \\
\text { fuentes históricas: documentos, } \\
\text { fotografias, figuras, mapas. } \\
\text { 5.Los estudiantes recuerdan } \\
\text { reinterpretan la Historia. } \\
\text { 6.Los estudiantes escuchan o ven films y } \\
\text { videos sobre historia. } \\
\text { 7.Uso textos escolares y algún otro } \\
\text { material (fotocopias). } \\
\text { 8.Participan en actividades grupales: } \\
\text { dramatizaciones, visitas a museos, } \\
\text { proyectos con la comunidad. }\end{array}$ \\
\hline $\begin{array}{l}\text { ¿En qué se concentran más sus clases de } \\
\text { Historia? }\end{array}$ & $\begin{array}{l}\text { 1.Procuro que conozcan los principales } \\
\text { hechos de la historia. } \\
\text { 2.Quiero que juzguen los principales } \\
\text { acontecimientos de la historia a partir del } \\
\text { punto de vista de los derechos humanos. } \\
\text { 3.Quiero que piensen el pasado tomando } \\
\text { en consideración todos los puntos de } \\
\text { vista. } \\
\text { 4.Quiero que comprendan el } \\
\text { comportamiento de las personas del }\end{array}$ \\
\hline
\end{tabular}




\begin{tabular}{|c|c|}
\hline & $\begin{array}{l}\text { pasado, reconstruyendo las formas de } \\
\text { vida y de pensamiento del periodo en que } \\
\text { esas personas vivieron. } \\
\text { 5.Quiero que usen la historia para } \\
\text { entender la situación del mundo actual y } \\
\text { descubrir las tendencias de cambio. } \\
\text { 6.Quiero que estudien historia de una } \\
\text { forma divertida y fascinante. } \\
\text { 7.Quiero que aprendan las tradiciones, } \\
\text { características, valores y misión de } \\
\text { nuestra nación y de nuestra sociedad. } \\
\text { 8.Quiero que aprendan a valorar los } \\
\text { vestigios históricos y las construcciones } \\
\text { antiguas }\end{array}$ \\
\hline $\begin{array}{l}\text { ¿Cuál de estos temas les interesan más a } \\
\text { los estudiantes? }\end{array}$ & $\begin{array}{l}\text { 1.La vida cotidiana y las personas } \\
\text { comunes. } \\
\text { 2.Reyes, presidentes y personajes } \\
\text { politicos importantes. } \\
\text { 3.Aventureros y grandes descubridores. } \\
\text { 4.Guerras, dictaduras y genocidios. } \\
\text { 5.Culturas de otros países lejanos. } \\
\text { 6.La formación de las naciones. } \\
\text { 7.El desarrollo de la democracia. } \\
\text { 8.La interferencia del hombre en el medio } \\
\text { ambiente. } \\
\text { 9.La historia de las relaciones de género. } \\
\text { 10.La historia de temas específicos (por } \\
\text { ejemplo, la historia de los automóviles, de } \\
\text { la iglesia, de la música, etc.) } \\
\text { 11.La historia de la familia del estudiante }\end{array}$ \\
\hline
\end{tabular}

Los datos obtenidos en las respuestas de los docentes, se analizaron a través del programa SPSS versión 22 para Mac. Se realizaron análisis de estadísticos descriptivos para cada ítem (media $(\mathrm{X})$, mediana $(\mathrm{Me})$, moda (M), mínimo y máximo), resultados que se compararon con la información de la investigación previa con los estudiantes. Para hallar diferencias significativas entre las respuestas de profesores y alumnos a los ítems del cuestionario, se empleó el test no-paramétrico de la $U$ de Mann-Whitney.

De forma complementaria y con el fin de matizar la comparación, se estableció el análisis de contenido de la planeación anual institucional del área de Ciencias Sociales para secundaria de los centros educativos en los que trabajaban los docentes que respondieron el cuestionario unidades de muestreo-. Se tuvo acceso a la información de diez de los trece colegios. Los items contemplados para cada una de las tres preguntas temáticas del cuestionario (Tabla 1), fueron utilizadas como las unidades de registro que guiaron el análisis de los objetivos educacionales propuestos, las temáticas formales, actividades y las mediaciones pedagógicas declaradas en las planeaciones. 


\section{Resultados}

\section{¿Qué ocurre normalmente en las clases de Historia?}

En relación a la primera pregunta, la cual estaba dirigida a identificar las concepciones de los docentes en torno a sus prácticas de enseñanza da la historia, la centralidad de la figura del profesor es evidente. Los items focalizados en la acción docente directa, tales como, oír sus explicaciones y obtener de ellos información sobre lo verdadero y falso en la historia, registran una frecuencia importante de repuestas asociadas a "frecuentemente" y "siempre". En el primer item, el consolidado de este tipo de respuestas se ubica en el $77,1 \%(n=47)$, mientras que en el segundo es del 65,6\% ( $\mathrm{n}=40)$. Asimismo, acciones direccionadas por el profesor que suponen la participación del estudiante, registran indicadores de respuesta similares. Por ejemplo, según los docentes, las discusiones conjuntas sobre diferentes explicaciones del pasado se realizan "frecuentemente" en un 60,7\% y, "siempre" en un $24,6 \%$ para un consolidado del $85,3 \%(n=52)$. En el caso del trabajo con fuentes históricas, dicha sumatoria alcanza el 60,7\% $(\mathrm{n}=37)$, mientras la participación de los estudiantes en diferentes actividades grupales registra un $54,1 \%(n=33)$. Estos resultados se diferencian de los datos obtenidos alrededor de la agencia directa de los estudiantes. De acuerdo al 50,8\% (n=31) de los docentes que participaron en la investigación, los estudiantes en sus clases solo " $a$ veces" recuerdan y reinterpretan la Historia, una cifra que supera ampliamente a la del profesorado que considera que esta acción se lleva a cabo frecuentemente $-27,9 \%$ - y siempre $-8,2 \%-$.

Al hacer un balance general de las respuestas de los docentes, se evidencia la presencia simultánea de métodos tradicionales y activos de enseñanza, por medio de los cuales, estos, caracterizan su práctica. Las explicaciones magistrales, el uso de fuentes históricas y la presencia de mediaciones pedagógicas como videos y films, se destacan. Sin embargo, al comparar esta información con las concepciones de sus estudiantes y las declaraciones de trabajo pedagógico contendidas en las planeaciones institucionales, se generan matices y diferencias considerables. Tales diferencias sugieren que la presencia de métodos activos y participativos de enseñanza-aprendizaje asociadas al desarrollo del pensamiento histórico no es tan fuerte como la mayoría de docentes creen.

Un primer indicador de esta situación se encuentra en la valoración que se hace sobre el principio de multiperspectividad y su desarrollo en las clases de Historia. Al comparar los resultados de las respuestas de docentes y estudiantes en torno al ítem número tres (Figura 1), el cual plantea la posibilidad de discutir diferentes explicaciones sobre lo que aconteció en el pasado, se identifica una diferencia significativa con 
$\mathrm{U}=15.461,5(\mathrm{p}=0.00<\mathrm{a}=0.05)$. Las respuestas de los estudiantes, a diferencia de las manifestadas por sus docentes, presentan valores asociados a "nunca" con un $2,1 \%(n=16)$ y "casi nunca" con un $12,4 \%$ $(\mathrm{n}=95)$. Asimismo, el porcentaje de la respuesta "a veces" es muy superior en la muestra de los estudiantes con un registro del 30,6\% ( $n=234)$, en comparación con el 14,8\% registrado por los docentes ( $n=9)$.

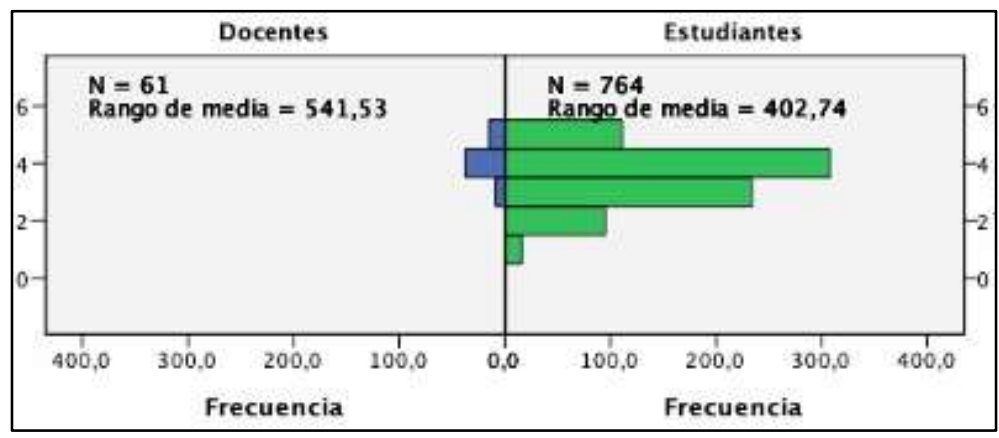

Figura 1. Prueba U de Mann-Whitney para muestras independientes ítem 3, pregunta ¿Qué ocurre normalmente en las clases de Historia

Por su parte, la contradicción entre lo manifestado por los docentes y las actividades propuestas en las planeaciones institucionales, es aún más aguda respecto a este ítem específico. Los datos obtenidos con el análisis de contenido de las planeaciones institucionales, indican una ausencia generalizada de tareas que implican, el reconocimiento de diversos posicionamientos y explicaciones sobre los fenómenos históricos que definen las temáticas desde las cuales se organizan los planes de estudio. De las 682 actividades de formación relacionadas con temáticas históricas, que fueron identificadas en los diez planes de estudio analizados, tan solo el $2,2 \%(n=15)$ de ellas contemplaban de alguna forma ejercicios de multiperspectividad.

En segundo lugar, aparece el ítem sobre el análisis de diversas fuentes históricas -ítem 4-, principio fundamental en el desarrollo de apuestas formativas que van más allá de la memorización de información. Aunque no existe una diferencia estadística significativa registrada en las respuestas de estudiantes y docentes en este ítem $(U=20.403$, $p=0,91$ > $\mathrm{a}=0.05$ ) (Figura 2), la presencia de fuentes en las planeaciones anuales de Ciencias Sociales, es muy débil y, no guarda una relación estrecha con el dato de la frecuencia de su empleo obtenida en la muestra de docentes $(M=4, X=3,69)$. Solo el 5,7\% $(n=27)$ de los objetivos formativos asociados a la enseñanza y el aprendizaje de la historia identificados en las planeaciones $(n=475)$ hacen referencia al uso, análisis o interpretación de fuentes históricas por parte de los estudiantes. 


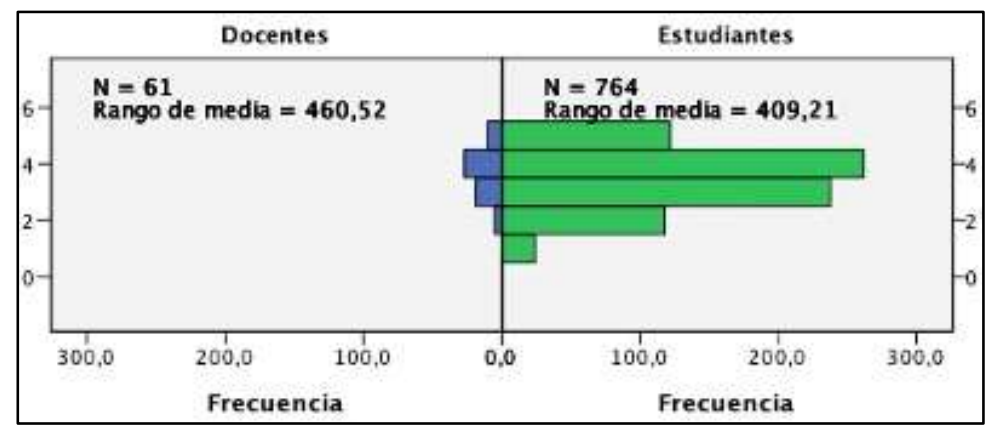

Figura 2. Prueba U de Mann-Whitney para muestras independientes ítem 4, pregunta ¿Qué ocurre normalmente en las clases de Historia

Además, de acuerdo a la información recolectada a través de las respuestas a los ítems de la pregunta uno, se presentan algunas diferencias entre las concepciones de docentes y alumnado alrededor de la implementación de otras mediaciones y estrategias pedagógicas en las clases de Historia. Según el 30,4\% de los estudiantes "nunca" (n=54) y "casi nunca" ( $\mathrm{n}=178)$ se tiene acceso a films y videos, mientras que solo el $8,2 \%(n=5)$ de los profesores reconocen esta ausencia. En este caso específico, la moda $(\mathrm{M})$ de las respuestas por parte de estos últimos $(M=4)$ es superior a la de sus alumnos $(M=3)$-se podría inferir entonces que existe una sobrevaloración en las concepciones de los maestros, de la presencia de estos medios en las aulas-. Una situación similar a la anterior se presenta en la valoración dada a la participación en actividades grupales, ítem que registra una $\mathrm{U}=7.581(\mathrm{p}=0.00<\mathrm{a}=0.05)$. Es importante tener en cuenta que, a pesar que las actividades grupales consignadas en las diez planeaciones analizadas representan el 22,4\% $(n=187)$ del total registrado $(n=682)$, el desarrollo de la mayoría, está concebido al interior del aula de clase; información que le daría más fuerza a los datos obtenidos en las respuestas del alumnado. Por otro lado, la valoración de los docentes y sus estudiantes alrededor de la presencia en las clases de historia de dispositivos pedagógicos como los textos escolares, cuyo uso tiende a ser negado o relativizado en el caso colombiano (Ibagón, 2019), presenta diferencias significativas $(\mathrm{U}=31.441,5, \mathrm{p}<0.05)$, hecho que permite pensar que el uso y circulación de este material es mayor a la reconocida por los maestros.

Finalmente, un dato significativo, resultado de la comparación entre las posturas de los docentes y sus estudiantes, está concentrado en los problemas identificados en torno a el papel del alumnado en la reinterpretación de la Historia. Los estudiantes son más críticos que los profesores frente a este tema. Un 39,9\% consideran que "nunca" (n=82) y "casi nunca" $(\mathrm{n}=232)$ se presenta esta acción en las clases, cifra que contrasta con el $13,1 \%$ de los docentes que respondieron a través de esta última opción ( $\mathrm{n}=8)$-una diferencia de 26,8 puntos porcentuales-. 


\section{¿En qué se concentran más las clases de Historia?}

Frente a los objetivos formativos que orientan las clases de Historia, las respuestas de los docentes presentan en la mayoría de items de la pregunta, medias superiores o cercanas a cuatro, con excepción de un solo ítem, el cual plantea la relación entre la enseñanza de la historia y los derechos humanos $(X=3,1)$ (Figura 3). Así pues, objetivos de aprendizaje relacionados con el conocimiento de hechos históricos -ítem 1 - $(X=4,15)$, la multiperspectividad histórica -ítem 3- $(X=4,1)$, la empatía histórica -ítem 4- $(X=4,31)$, el cambio y la continuidad -ítem 5- $(X=4,52)$, el gusto por el aprendizaje -ítem 6- $(X=4,23)$, la identidad nacional -ítem 7 - $(X=3,95)$ y, la valoración del patrimonio -ítem 8 - $(X=3,92)$, según los docentes, se desarrollan "frecuentemente" y "siempre" con escasas frecuencias registradas en nunca y "casi nunca". En este sentido, desde su perspectiva, las clases de historia están pensadas desde una integración de objetivos tradicionales y renovadores de aprendizaje histórico, que son valorados positivamente en una escala de importancia similar (Figura 3).

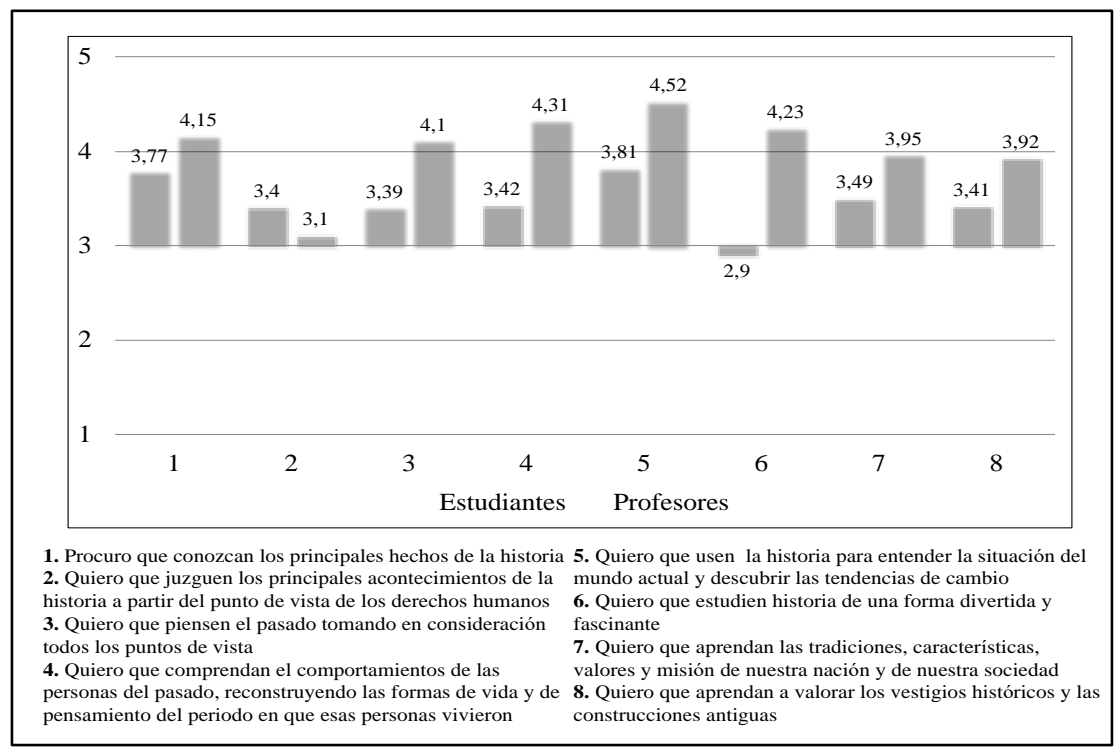

Figura 3. Medias en las respuestas de estudiantes y docentes a los items de la pregunta ¿En qué se concentran más las clases de Historia?

No obstante, el ejercicio comparativo entre esta información y las concepciones de los estudiantes frente a los mismos temas, nuevamente marca diferencias estadísticas significativas que, se amplian respecto a las identificadas en la pregunta sobre las prácticas y procedimientos de enseñanza y aprendizaje de la historia -pregunta 1-. Aunque al aplicar 
la prueba de la U de Mann-Whitney en todos los ítems de la pregunta dos se rechaza la hipótesis nula $\left(\mathrm{H}_{0}\right) \quad(\mathrm{p}<0.05)$, las diferencias significativas son más marcadas en los items que contemplan racionalidades vinculadas al desarrollo del pensamiento histórico. Por ejemplo, en el caso del objetivo formativo de la multiperspectividad histórica -ítem3- las disimilitudes en las frecuencias registradas para "frecuentemente" y "siempre" son más bajas en los estudiantes $(49,3 \%$, $\mathrm{n}=376)$ en comparación a los docentes $(80,3 \%, \mathrm{n}=49)$ - Una situación que se repite en los ítems sobre la empatía histórica y el cambio histórico con una diferencia de 37,6 y 31,2 puntos porcentuales respectivamente. Asimismo, mediante el análisis de las declaraciones contenidas en las planeaciones anuales quedan de manifiesto una serie de vacíos entre la valoración que hacen los docentes sobre los objetivos formativos enfocados en el desarrollo del pensamiento histórico y, lo que oficialmente queda consignado en sus propias rutas institucionales de trabajo pedagógico. De los 475 objetivos identificados relacionados con la enseñanza y el aprendizaje de la historia, el 1,9\% (n=9) está relacionado con la multiperspectividad, el 3,3\% $(n=16)$ con la empatía histórica, y el 18,3\% ( $n=87)$ con el cambio histórico; cifras muy bajas si se tiene en cuenta lo manifestado por los docentes en sus respuestas al cuestionario -el mayor porcentaje se concentra en la caracterización de contenidos sustantivos de la historia $(55,5 \%, \mathrm{n}=264)$ -

Otro de los temas en los que se identifican diferencias significativas entre las concepciones de docentes y estudiantes sobre los objetivos de las clases de historia, es en el hecho de que estas últimas sean interesantes y divertidas -ítem 6- $(\mathrm{U}=7.226,5, \mathrm{p}<0.05)$. Mientras el $88,6 \%$ de los docentes consideran que "frecuentemente" $(52,5 \%, n=32)$ y "siempre" $(36,1 \%, n=22)$ sus clases tienen dicho fin, solo el $27 \%$ de sus estudiantes $(n=206)$ lo perciben de esta forma (Figura 4). En contraste el $33,5 \%$ de los alumnos consideran que "nunca" $(9,3 \%, n=71)$ y "casi nunca" $(24,2 \%, n=185)$ se cumple con este item; opciones de respuesta que en el caso de los docentes se ubica en el 1,6\% ( $n=1)$.

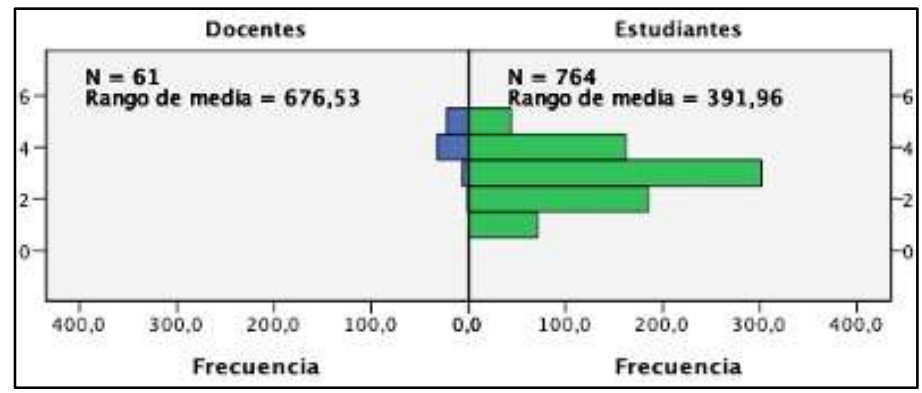

Figura 4. Prueba U de Mann-Whitney para muestras independientes item 6, pregunta ¿En qué se concentran más las clases de Historia? 


\section{¿Cuáles de estos temas les interesan más a sus estudiantes?}

Respecto al interés de los estudiantes por determinados contenidos sustantivos de enseñanza de la historia, las respuestas de los docentes a los items de esta pregunta, presentan medias inferiores a las registradas en los ítems de las preguntas 1 y 2 -salvo algunas excepciones-. En este sentido, sus posicionamientos son más cautos y en algunos casos se asocian con valores negativos (Figura 5).

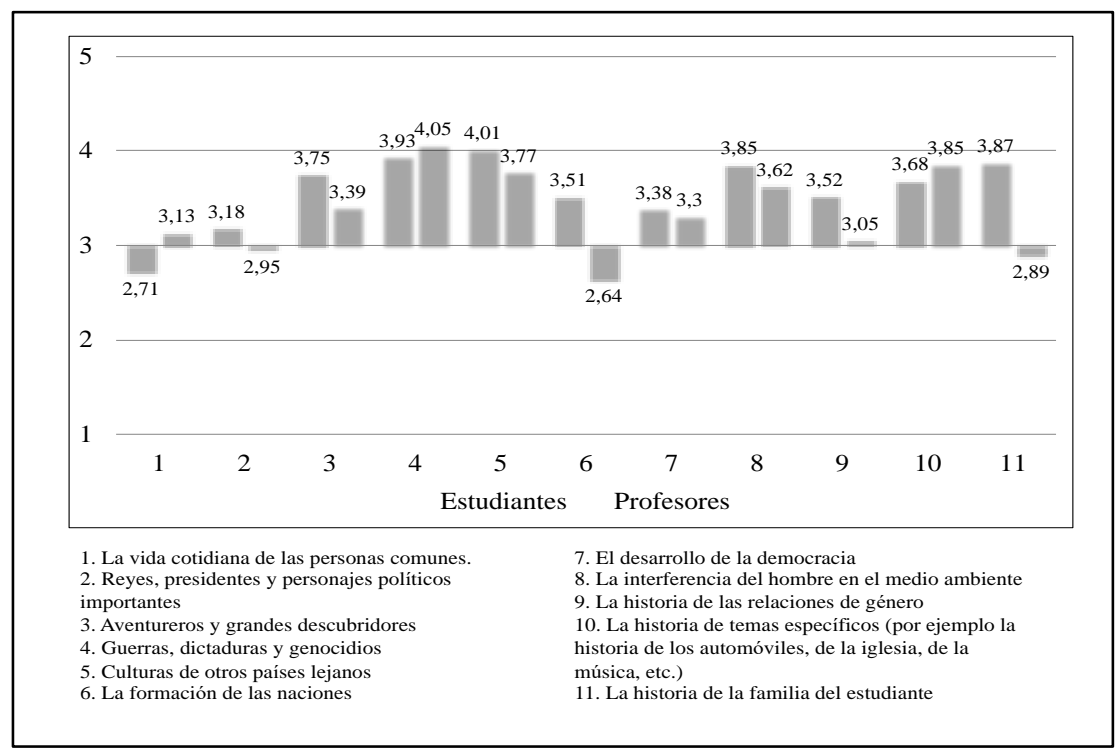

Figura 5. Medias en las respuestas de estudiantes y docentes a los ítems de la pregunta ¿Cuáles de estos temas les interesan más a sus estudiantes?

De los once ítems vinculados a esta pregunta, tres presentan medias de respuesta por debajo de tres (3): item 3, "Reyes, presidentes y personajes políticos importantes" $(X=2,95)$; ítem 6 , "La formación de las naciones" $(X=2,64)$ e; item 11 , "La historia de la familia de los estudiantes" $(X=2,89)$. La única media que se encuentra por encima de cuatro (4) es la del ítem 4, el cual está asociado a las temáticas de "Guerras, dictaduras y genocidios" $(X=4,05)$. Los demás ítems registran valores que oscilan entre 3 y 4 (Figura 5 ).

Lo interesante al contrastar estos datos con el análisis de la frecuencia de la mención de dichos contenidos sustantivos en las planeaciones institucionales anuales del área de Ciencias Sociales, es que el ítem de "La formación de las naciones" cuya media es la más baja de la totalidad de ítems de todo el cuestionario de docentes, de las once temáticas generales, es la que está más presente en estos documentos 
curriculares $^{3}$. Así pues, de 549 contenidos sustantivos identificados, 168 $(30,6 \%)$ tienen relación directa con este ítem. Le siguen, "Culturas de otros países lejanos" $(19,6 \%, \mathrm{n}=108)$ y "Guerras, dictaduras y genocidios" $(19,1 \%, n=105)$. El menor porcentaje registrado es el de "La historia de la familia del estudiante" con un $0,9 \%(n=5)$.

En contraste con las diferencias significativas analizadas entre las concepciones de estudiantes y docentes frente a los ítems de las dos primeras preguntas, la mayoria de desigualdades identificadas alrededor de los intereses temáticos del alumnado, se da por una mayor valoración de los estudiantes de algunos de estos items en comparación a la otorgada por los docentes -salvo el ítem 1, "La vida cotidiana de las personas comunes" (Figura 5)-. De este grupo, resaltan especialmente las diferencias significativas halladas en los ítems número once $(\mathrm{U}=34.952,5, \mathrm{p}<0.05)$ y seis $(\mathrm{U}=34.300,5, \mathrm{p}<0.05)$-los temas con menor y mayor porcentaje de presencia en las planeaciones anuales-. En el primer caso, el $67,7 \%$ de los estudiantes manifestó tener "mucho interés" $(34,2 \%, n=261)$ e "interés total" $(33,5 \%, n=256)$ en la historia de su familia, una cifra mayor a la consolidada en las respuestas de los docentes, la cual se ubica en el 27,9\% (n=17) (Figura 6). En el segundo caso, tan solo el $13,1 \%(\mathrm{n}=8)$ de los docentes estableció que sus estudiantes tienen "mucho interés" $(8,2 \%, n=5)$ e "interés total" $(4,9 \%$, $\mathrm{n}=3$ ) en la formación de las naciones, cifra que se distancia 40,4 puntos porcentuales del total de la frecuencia de respuestas que indican "mucho interés" $(37 \%, \mathrm{n}=283)$ e "interés total" $(16,5 \%, \mathrm{n}=126)$ de parte de los alumnos por esta temática.

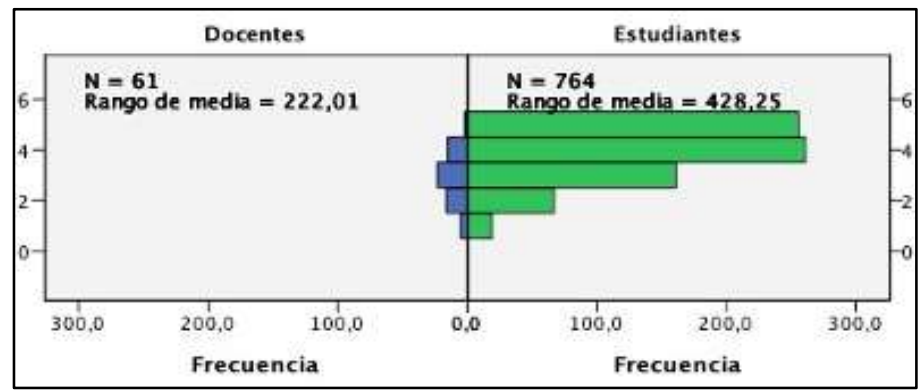

Figura 6. Prueba U de Mann-Whitney para muestras independientes ítem 6, pregunta ¿En qué se concentran más las clases de Historia?

Por último, es importante destacar que no se encontraron diferencias significativas en tres de los once items de esta pregunta: ítem 4, "Guerras, dictaduras y genocidios" $(\mathrm{U}=22.224, \mathrm{p}=0,535>\mathrm{a}=0.05)$; item

\footnotetext{
${ }^{3}$ Es importante tener en cuenta que, debido a su naturaleza, en un contenido se pueden encontrar varios ítems relacionados. Por esta razón el porcentaje final de frecuencia de los ítems excede el $100 \%$.
} 
7, "El desarrollo de la democracia" ( $U=25.147, p=0,276>\mathrm{a}=0.05)$ e; ítem 10 , "La historia de temas específicos" ( $U=21.663,5, p=0,342>a=0.05)$. De estos resultados se puede inferir que los docentes en general logran identificar con relativa claridad el interés real de sus estudiantes frente a estas temáticas, algo que no sucede alrededor de los demás temas propuestos, lo cual supone un problema pedagógico importante.

\section{Discusión y conclusiones}

El análisis de las respuestas de los docentes en torno a los ítems referidos a los núcleos de indagación sobre tipo de prácticas y objetivos formativos de las clases de historia, deja de manifiesto la convergencia que existe entre intereses y nociones de trabajo pedagógico de carácter crítico - vinculadas el desarrollo del pensamiento histórico- y tradicional -vinculadas a discursos románticos y procedimientos técnicos de enseñanza-. Aunque los primeros son por lo general mejor valorados no existen mayores disparidades estadísticas con los segundos. Este resultado en principio, establece una diferencia con hallazgos de investigaciones que ubican las concepciones o representaciones sociales sobre la enseñanza de la historia de profesores en ejercicio y en formación, en alguno de los dos extremos, ya sea, el definido por las perspectivas críticas (Gago, 2018; González et al, 2020; Voet \& De Wever, 2016) o, por las tradicionales (Yilmaz, 2008).

Sin embargo, la comparación realizada entre las concepciones de los docentes y sus estudiantes, matiza dichos resultados, en la medida que, para estos últimos, la experiencia formativa al interior de las aulas de clase está definida principalmente a partir de perspectivas de corte tradicional. Por consiguiente, tanto, las formas de trabajo pedagógico mediante las cuales los docentes caracterizan sus prácticas de enseñanza de la historia, como, los vínculos que establecen entre estas y la búsqueda del cumplimiento de objetivos de formación sustentados en el desarrollo del pensamiento histórico, no están conectadas del todo con lo que finalmente perciben y entienden sus estudiantes. Dicha situación, se vuelve aún más problemática, si se tiene en cuenta que las concepciones de los docentes no se ven reflejadas en lo que ellos mismos registran al interior de sus planeaciones; documentos en los que es dificil encontrar sustento explicito de actividades y metas de formación basadas en contenidos meta-históricos.

En este sentido, los resultados evidencian que, a pesar que existe un reconocimiento por parte de los profesores de Ciencias Sociales de la importancia de desarrollar las clases de historia a partir de procesos formativos que van más allá de la reproducción 
y memorización de información, existen problemas significativos en los mecanismos de concreción de este ideal en la práctica. Sus mensajes e intencionalidades educacionales, no están logrando ser reconocidos integralmente por sus estudiantes, condición que es central para impactar positivamente los aprendizajes de estos.

Así pues, la distancia entre lo que aspira a realizar el docente en torno a la formación histórica de sus estudiantes y lo que finalmente se materializa en las clases -conforme la perspectiva del estudiantado-, ratificaría los vacíos que han señalado algunos autores alrededor de la fundamentación específica en didáctica de la historia del profesorado de ciencias sociales e historia (Andelique, 2011; Ibagón, 2016; Miralles, Sánchez, \& Arias, 2014). Debido a su naturaleza, el desconocimiento de los docentes de ciencias sociales en torno a la didáctica de la historia, entendida como ciencia del aprendizaje histórico (Rüsen, 2007; Schmidt, 2019), explicaría gran parte de las dificultades que se presentan al interior de las aulas de clase al momento de desarrollar ejercicios basados en la cognición histórica situada -que sean reconocidos, valorados e interiorizados por los alumnos-, así como, los problemas detectados alrededor del proceso de identificación, por parte de los docentes, de los intereses de aprendizaje de sus alumnos.

En esta medida, es fundamental reconocer que ante el peso considerable que tiene la enseñanza de la historia en el currículo de ciencias sociales colombiano -a pesar de su enfoque integracionista-, seguir negando la importancia de pensar teóricamente y metodológicamente la didáctica de la historia en tanto campo específico de formación inicial y permanente de los docentes del área, facilita la reproducción de discursos y prácticas educativas limitadas, que terminan por reducir a su mínima expresión las potencialidades educacionales del aprendizaje histórico en la escuela. Por lo tanto, en los procesos de transformación de los sentidos y alcances de la enseñanza de la historia respecto a sus pautas tradicionales, no es suficiente para su concreción, la introducción de "innovaciones" educativas, si en el fondo se desconoce la fundamentación epistémica de estas. Por ejemplo, emplear en el aula fuentes sin que estas se configuren y entiendan como evidencia histórica, solo encubre la predominancia de métodos y horizontes educativos tradicionales de corte memorístico (Cowgill \& Waring, 2017); permanencias que son percibidas por el alumnado.

Asimismo, desarrollar procesos formativos en historia, desconociendo los posicionamientos de los estudiantes en torno a estos, afecta la toma de decisiones frente a la práctica docente. De 
ahí que, es importante que los profesores analicen y evalúen continuamente, si los objetivos de formación con sus estudiantes se están cumpliendo $\mathrm{y}$, en esta medida, si las lógicas de las actividades que han dispuesto para ello, están siendo comprendidas e interiorizadas por los alumnos.

Aunque siempre existirá un margen de error entre lo que proyecta el docente y lo que finalmente termina apropiando el estudiante, la diferencia se puede acortar a través del reconocimiento sistemático de las ideas, concepciones e intereses del alumnado, no solo, en torno a contenidos específicos, sino a la vez, alrededor de las mediaciones pedagógicas por medio de las cuales se abordan estos; tarea en la que la didáctica de la historia es base fundamental e imprescindible.

\section{Referencias}

Acevedo, A. y Samacá, G. (2012). La política educativa para la enseñanza de la Historia de Colombia (1948-1990): de los planes de estudio por asignaturas a la integración de las ciencias sociales. Revista Colombiana de Educación, (62), 221-244. doi: $10.17227 / 01203916.1637$

Aguilera, A. (2017). La enseñanza de la historia y las ciencias sociales hoy: contrasentidos y posibilidades. Folios, (46), 15-27.

Amézola, G. \& Cerri, L. (Coord.) (2018). Los jóvenes frente a la Historia. Aprendizaje y enseñanza en escuelas secundarias. La Plata: Facultad de humanidades y Ciencias de la Educación, Universidad de la Plata.

Andelique, C. (2011). La didáctica de la Historia y la formación docente: ¿Qué profesor de historia necesitan las escuelas? Clio \& Asociados, 15, 256-269.

Angvik, M. \& Borries, B. (1997). Youth and History: a comparative European survey on historical consciousness and political attitudes among adolescents. Hamburg: Körber-Stiftung.

Arias, D. (2015). La enseñanza de las ciencias sociales en Colombia: Lugar de las disciplinas y disputa por la hegemonía de un saber. Revista de Estudios Sociales, (52),134-146.

Arias, D. (2017). Relatos de Nación y Escuela. Colombia en los imaginarios de docentes en formación. Bogotá: Universidad Distrital "Francisco José de Caldas".

Barca, I. (2019). Narrativas históricas de los jóvenes: una cara de su orientación temporal. Historia y Espacio, 15(53), 309-332. doi: 10.25100/hye.v15i53.8741.

Borries, B. (2011). Historical Consciousness and historical learning: 
some results of my own empirical research. In L. Perikleous \& D. Shemilt (Ed.). The future of the past: Why History Education Matters (pp. 283-319). Cyprus: AHDR/UNDP_ACT.

Cerri, L. (Org.) (2018). Os jovens e a história: Brasil e América do sul. Ponta Grossa: Ed. UEPG.

Cowgill, D., \& Waring, S. (2017). Historical thinking: An evaluation of student and teacher ability to analyze sources. Journal of Social Studies Education Research, 8(1), 115-145.

Gago, M. (2016). Consciência histórica e narrativa no ensino da História: lições da História? Ideias de professores e alunos de Portugal. Revista História Hoje, 5(9), 76-93.

Gago, M. (2018). Ser Professor de História em tempos difíceis: início de um processo formativo. Antiteses, 11(22), 505-515. doi: 10.5433/1984-3356.2018v11n22p507.

Gómez, J. (2015). Las ciencias sociales escolares: entre la realidad y el deseo. Voces y silencios. Revista Latinoamericana de Educación, 6(2), 101-113.

González, M. (2018). La historia escolar y los profesores. Una mirada desde el Mercosur. En Amézola, G., \& Cerri, L. (Coord.). Los jóvenes frente a la Historia. Aprendizaje y enseñanza en escuelas secundarias (pp. 153-182). La Plata: Facultad de humanidades y Ciencias de la Educación, Universidad de la Plata.

González, G., Santisteban, A., \& Pagès, J. (2020). Finalidades de la enseñanza de la historia en futuros profesores. Magis, Revista Internacional de Investigación en Educación, (13), 1-23. doi: 10.11144/Javeriana.m13.fehf

Guerrero, C. (2011). La enseñanza de la Historia en el contexto de las Ciencias Sociales en Colombia: de la tecnología educativa a las inteligencias múltiples (1970-2008). En J, Guerrero \& L. Weisner (Comp.). ¿Para qué enseñar Historia? (pp. 189-208). Medellin: UPTC/La Carreta Editores.

Ibagón, N. (2016). Enseñar y aprender Historia a partir del análisis de fuentes históricas. Una experiencia formativa en Educación Superior. Voces y silencios. Revista Latinoamericana de Educación, 7 (1), 121-133. doi:10.18175/vys7.1.2016.06

Ibagón, N. (2019). Una historia dificil de narrar. El periodo de La Violencia en los textos escolares de Ciencias Sociales. Cali: Programa Editorial Universidad del Valle.

Ibagón, N., \& Minte, A. (2019). El pensamiento histórico en contextos escolares. Hacia una definición compleja de la enseñanza de Clío. Zona Próxima, 31, 107-131. doi: 10.14482/zp.31.370.7

Kitson, A., Steward, S. \& Husbands, C. (2015). Didáctica de la historia en secundaria obligatoria y bachillerato. Madrid: Morata.

McCully, A. (2012). History Teaching, Conflict and the Legacy of the Past. 
Education, Citizenship and Social Justice, 7(2), 145-59.

Miralles, P., Prats, J. y Tatjer, M. (2012). Conocimientos y concepciones de estudiantes españoles y latinoamericanos de educación secundaria obligatoria sobre las independencias politicas americanas. Scripta Nova Revista Electrónica de Geografía y Ciencias Sociales, 16 (418), 1-12.

Miralles, P., Sánchez, R., Arias, L. (2014). La formación profesorado en didáctica de la historia: modelos de formación y propuestas para el desarrollo profesoral. Perspectiva, 32(2), 497-519.

Monteagudo, J., Molina, S., \& Miralles, P. (2015). Opiniones sobre evaluación de los profesores de segundo ciclo de ESO de Geografia e Historia en España. El caso de la región de Murcia. Revista Mexicana de Investigación educativa, 20(66), 737-761.

Padilla, A. \& Bermúdez, Á. (2016). Normalizar el conflicto y desnormalizar la violencia: retos y posibilidades de la enseñanza crítica de la historia del conflicto armado colombiano. Revista Colombiana de Educación, 71, 219-251.

Rodríguez, S. (2013). Aproximación a la formación de profesores en ciencias sociales y en historia en Colombia: elementos para el debate. Reseñas de enseñanza de la Historia, (10), 29-56.

Rüsen, J. (2007). História Viva. Teoria da História III: formas e funções do conhecimento histórico. Brasilia: Unb.

Schmidt, M. (2019). El turno de la didáctica de la historia: contribuciones para un debate. Historia y Espacio, 15(53), 21-42. doi: 10.25100/hye.v15i53.8734.

Vargas, S., Acosta, M., \& Sánchez, R. (2013). Historia, memoria, pedagogia: Una propuesta alternativa de enseñanza/aprendizaje de la historia. Bogotá: Universidad Minuto de Dios.

Voet, M., \& De Wever, B. (2016) History Teachers' Conceptions of Inquiry-Based Learning, Beliefs about the Nature of History, and their Relation to the Classroom Context. Teaching and Teacher Education, 55, 57-67. doi: 10.1016/j.tate.2015.12.008

Yilmaz, K. (2008). Social Studies Teachers' Conceptions of History: Calling on His- toriography. The Journal of Educational Research, 101 (3), 158-176. doi: 10.3200/JOER.101.3.158-176 